\title{
Salud y calidad de vida de cuidadores familiares y profesionales de personas mayores dependientes: estudio comparativo
}

\author{
Noelia Flores ${ }^{1}$, Cristina Jenaro ${ }^{1}$, Lourdes Moro ${ }^{1}$ y Raluca Tomşa ${ }^{2}$ \\ ${ }^{1}$ Universidad de Salamanca (España); ${ }^{2}$ Universitatea din Bucuresti (Romania)
}

\begin{abstract}
El trabajo que se presenta tiene como objetivo analizar las semejanzas y diferencias en la salud y en la calidad de vida de los cuidadores familiares y profesionales que atienden a personas mayores en situación de dependencia. Concretamente se presentan los resultados obtenidos tras la evaluación de 600 cuidadores de los cuales un 33,83\% son familiares y un $66,17 \%$ son profesionales, a quienes se evaluó la carga o estrés experimentado, la salud física y psicológica y la calidad de vida general, laboral, familiar y social. Los resultados obtenidos indican que existen diferencias entre ambos grupos de cuidadores. Los familiares dedicados al cuidado presentan mayores niveles de sobrecarga y tienen una peor salud física y psicológica como consecuencia de la atención prestada al dependiente. Los cuidadores profesionales experimentan una mayor satisfacción con la vida en general y con otros factores como el trabajo, la familia y las relaciones sociales. Finalmente se justifica la necesidad de poner en marcha intervenciones de mejora de la salud de los cuidadores.
\end{abstract}

Palabras clave: Cuidadores, sobrecarga, salud, calidad de vida, dependencia.

Health and quality of life of family and professional caregivers of dependent elderly: a comparative study. The work presented aims to analyze the similarities and differences in health and quality of life of family caregivers and professionals serving seniors in a position of dependency. Specifically, the results presented were obtained from the evaluation of 600 caregivers, of which $33.83 \%$ are family and $66.17 \%$ are professionals, to determine their burden or stress experienced, as well as their physical and psychological health and their overall, work, family and social quality of life. The results indicate that there are differences between the two groups of caregivers. The family caregivers have higher levels of overload and have poorer physical and psychological health as a result of their tasks. Professional caregivers experience greater satisfaction with life in general and in other factors such as work, family and social relationships. Finally, this justifies the need to implement interventions to improve the health of caregivers.

Keywords: Caregivers, overload, health, quality of life, dependence.

Correspondencia: Noelia Flores. Facultad de Psicología. Universidad de Salamanca. Avda. de la Merced, 109-131. C.P.: 37005. Salamanca (España). E-mail: nrobaina@usal.es 
En las últimas décadas se ha observado un cambio demográfico importante. Los índices de natalidad han disminuido y la esperanza de vida está aumentando cada vez más, pasando de los 35 años, a principios del siglo XX, a los 50 años a mediados de éste, y superando los 78 años en la actualidad. Ello ha generado una población mayor cada vez más envejecida. Las causas de esto las podemos encontrar en los grandes avances experimentados por la medicina y en los cambios sociales acaecidos a lo largo de todos estos años. Todo ello conlleva que las personas cada vez sean más longevas y que se produzca, a su vez, un sobre envejecimiento de la población más mayor. Ley 39/2006, de 14 de diciembre de Promoción de la Autonomía Personal y Atención a las personas en situación de dependencia, entiende por dependencia el "estado de carácter permanente en que se encuentran las personas que, por razones derivadas de la edad, la enfermedad o la discapacidad, y ligadas a la falta o a la pérdida de autonomía física, mental, intelectual o sensorial, precisan de la atención de otra u otras personas o ayudas importantes para realizar actividades básicas de la vida diaria o, en el caso de las personas con discapacidad intelectual o enfermedad mental, de otros apoyos para su autonomía personal".

Esta definición, que ha sido ampliamente aceptada, plantea la concurrencia de tres factores: en primer lugar, la existencia de una limitación física, psíquica o intelectual que merma determinadas capacidades de la persona; en segundo lugar, la incapacidad de la persona para realizar por sí mismo las actividades de la vida diaria; y en tercer lugar, la necesidad de asistencia o cuidados por parte de un tercero. Las personas dependientes, por tanto, van a requerir de una intervención continuada, aunque no necesariamente permanente, basada en el apoyo y los cuidados. De ahí la importancia de los cuidados formales e informales en la atención a la necesidades de este colectivo. La familia es la principal fuente de ayuda para las personas mayores y adquiere aún más importancia cuando éstas sufren algún tipo de dependencia pues contribuye a rellenar las lagunas o carencias de las redes sociosanitarias de servicios y constituye un eje fundamental de las políticas sociales basadas en el "envejecimiento en el hogar". Así mismo, también la familia contribuye a mantener la seguridad, la calidad de vida y el bienestar tanto físico como psicológico de la persona mayor dependiente (Crespo y López, 2007; Fernández de Larrinoa et al., 2011; Ruíz-Robledillo y Moya-Albiol, 2012). Este tipo de cuidado que da la familia se suele conocer también como cuidado informal. El Libro Blanco (IMSERSO, 2005, p. 173) lo define como el cuidado que se presta por parientes, amigos o conocidos. Más concretamente, se puede decir que es la atención no remunerada que se presta a las personas con algún tipo de dependencia por parte de los miembros de su familia y/u otra persona sin otro lazo de unión ni obligación con la persona dependiente que no sea el de la amistad o el de la buena vecindad (Rivera, 2001). Así, cuando alguien enferma o presenta algún tipo de dependencia, dentro del sistema familiar suele haber una persona, el cuidador principal, que asume las tareas de cuidado básicas, y que suele 
ser percibida por los restantes miembros de la familia como el responsable de asumir el cuidado del enfermo (Feldberg et al., 2011; Fernández de Larrinoa et al., 2011). Numerosos estudios coinciden en afirmar que el perfil del cuidador principal se corresponde con el de una mujer, de edad comprendida entre los 45 y 69 años, que informa no recibir la ayuda de nadie para realizar este trabajo y que se caracteriza mayoritariamente por no tener una actividad laboral retribuida (Crespo y López, 2007; Flores, Jenaro, Navarro, Vega y Vázquez, 2013; Grandón, Jenaro y Lemos, 2008).

Por su parte, el cuidado formal alude al "conjunto de acciones que un profesional oferta de forma especializada, y que va más allá de las capacidades que las personas poseen para cuidar de sí mismas o de los demás" (Francisco del Rey y Mazarraza, 1995, p. 62). Se trata, fundamentalmente, de toda aquella asistencia a personas mayores que necesitan ayuda en las actividades de la vida diaria, y en la que concurre alguno de los siguientes criterios: el cuidado se provee sobre una relación contractual (oral o escrita) o a través de una organización (empresa, institución pública u organización sin ánimo de lucro). Por tanto, el proveedor de cuidado formal es un profesional que oferta un servicio para el que tiene una preparación que lo habilita (Rogero, 2010). En este sentido, desempeña tareas de asistencia especializada, a diferencia de la atención generalista que se le atribuye al cuidador familiar. Por otro lado, la relación entre cuidador profesional y receptor está caracterizada por una neutralidad afectiva, en contraste con la implicación emocional del cuidador familiar (Herrera-Gómez, 1998). Pese a ello, es necesario destacar el papel protagonista que estos trabajadores tienen para garantizar una atención de calidad, dado que después de la familia, proveen una mayor y más intensa atención a la persona dependiente (Aparicio et al., 2008).

Existe un amplio consenso respecto a las repercusiones negativas del cuidado en la salud y calidad de vida del cuidador familiar (Crespo y López, 2007; Flores et al., 2013; Grandón, Jenaro y Lemos, 2008). La mayoría de los estudios constatan que la presión a la que están expuestos los cuidadores puede desencadenar en ellos sentimientos de estrés, ansiedad y depresión (Hartke, King, Heinemann y Semik, 2006). Otros autores también informan que el cuidado afecta negativamente a la salud física y psicológica (Brummett et al., 2006). Por tanto, está ampliamente contrastado que cuidar de una persona mayor dependiente es una experiencia estresante que puede llegar a cronificarse $\mathrm{y}$ tener repercusiones negativas para la persona proveedora del cuidado, especialmente en aquellos casos en que el cuidador perciba la situación como altamente demandante y los cuidados se ofrezcan de manera continuada (ocupando gran parte del tiempo del cuidador) y prolongada en el tiempo. Por otro lado, respecto a los cuidados formales, es preciso aludir al concepto de carga experimentada y más concretamente al estrés laboral de tipo crónico o síndrome de burnout. En este sentido, numerosas investigaciones sobre el síndrome de quemarse por el trabajo ponen de manifiesto su repercusión en variables 
psicológicas, fisiológicas, organizacionales y sociales que inciden en el individuo provocándole alteraciones emocionales y conductuales de diversa índole (Maslach, Schaufeli y Leiter, 2001). También se ha constatado las repercusiones del estrés crónico en la salud física y emocional de los cuidadores formales (Jenaro, Flores y Arias, 2007; Menezes de Lucena, Fernández, Hernández, Ramos y Contador, 2006). El contacto con el usuario es la base de estos trabajos y dicho contacto suele ser bastante intenso y, en ocasiones, poco gratificante puesto que se deposita en el profesional una gran carga de responsabilidad que podrá provocar su propio desbordamiento con la consecuente pérdida de capacidades y de motivación por su trabajo (Jenaro, Flores y Arias, 2007).

Pese a esto, en nuestro país no existen muchos estudios comparativos que hayan analizado las principales semejanzas y diferencias en la salud y calidad de vida experimentada como consecuencia de los cuidados de personas mayores dependientes en los colectivos de cuidadores familiares y profesionales. Por tanto, y a la vista de lo anteriormente expuesto, el presente trabajo pretende contribuir a incrementar los conocimientos referentes a las necesidades de los cuidadores familiares y profesionales que atienden a personas mayores en situación de dependencia y aportar datos que permitan la puesta en marcha de intervenciones que mejoren la salud de este colectivo. Más concretamente, con el presente trabajo pretendemos dar respuesta a los siguientes objetivos: (1) conocer el nivel de carga o estrés experimentado por los cuidadores familiares y profesionales de personas mayores dependientes; (2) determinar semejanzas y diferencias en la salud física y psicológica de los cuidadores y (3) identificar semejanzas y diferencias en la calidad de vida experimentada por los cuidadores.

\section{MÉTODO}

\section{Participantes}

Los participantes, una muestra de 600 cuidadores de personas mayores en situación de dependencia, se distribuyen en 203 (33,83\%) cuidadores familiares y 397 $(66,17 \%)$ cuidadores profesionales. Del total, 39 (6,5\%) se caracterizan por ser hombres frente a $561(93,5 \%)$ mujeres. La edad promedio de los participantes es de 48 años $(D T=11,13)$. De los 600 participantes, $429(71,5 \%)$ indicaron estar casados o conviviendo en pareja, $105(17,5 \%)$ son solteros, $36(6 \%)$ están divorciados y/o separados y $28(4,7 \%)$ indicaron ser viudos/as. Tan sólo dos cuidadores $(0,3 \%)$ no ofrecieron información respecto a su estado civil. Por otro lado, 410 participantes $(68,3 \%)$ manifestaron tener cargas familiares, esto es, hijos $\mathrm{u}$ otros familiares conviviendo y dependiendo económicamente de ellos frente a 185 (30,8\%) que no presentan dichas cargas. Respecto al tiempo que llevan desempeñando la función de cuidador cabe destacar cómo la mayor parte de los cuidadores profesionales $(41 \%)$ llevan entre 3 y 8 años trabajando como cuidadores, mientras que en el grupo de 
cuidadores familiares predominan $(29,6 \%)$ quienes llevan más de 11 años atendiendo a su familiar. De forma específica, los cuidadores familiares se caracterizan por ser fundamentalmente mujeres $(83,7 \%)$, hijas $(59,1 \%)$ y/o esposas $(21,2 \%)$ de la persona atendida que suele ser una persona mayor cuya causa principal de dependencia tiene que ver mayoritariamente con las demencias (Alzheimer, demencia senil, demencia de los cuerpos de Lewis, etc.) y la enfermedad mental (esquizofrenia), respectivamente. Así mismo, los cuidadores profesionales también se caracterizan por ser mayoritariamente mujeres $(98,5 \%)$ que desempeñan su empleo en el sector de la ayuda a domicilio y que atienden fundamentalmente a personas mayores en situación de dependencia (97\%).

\section{Instrumentos}

Para el presente estudio se utilizaron dos cuestionarios ampliamente conocidos, como son el Cuestionario de Carga del Cuidador (CBI) y el Cuestionario de Salud de Goldberg, versión de 28 ítems (GHQ-28). Además, se diseñó un cuestionario para la evaluación de variables sociodemográficas y contextuales relacionadas con el cuidado. El CBI de Zarit, Reever y Bach-Peterson (1980) se utilizó para evaluar la carga subjetiva experimentada por los cuidadores. Se trata de un instrumento de 22 ítems que se puntúan en una escala tipo Likert de 5 puntos que va desde "nunca" (0) a "casi siempre" (4). La puntuación total se obtiene sumando las puntuaciones de cada uno de los ítems y oscila entre 0 y 88. Puntuaciones más altas indican un mayor grado de sobrecarga. Así, puntuaciones inferiores a 45 son indicativas de ausencia de sobrecarga. Por su parte, puntuaciones comprendidas entre 46 y 55 indican sobrecarga leve, y puntuaciones superiores a 56 son indicativas de sobrecarga intensa. El cuestionario CBI es el único instrumento adaptado a la población española para evaluar la carga subjetiva. Posee, además, buenas propiedades psicométricas. La fiabilidad del instrumento fue contrastada mediante el alfa de Cronbach y los resultados (alfa=0,92) apoyaron su fiabilidad. En segundo lugar utilizamos la adaptación española (Lobo, Perez y Artal, 1989) de la versión de 28 ítems del General Health Questionnaire GHQ-28 (Goldberg y Hillier,1979) para evaluar la salud física y psicológica. La presente versión consta de cuatro subescalas: quejas somáticas, disfunción social, ansiedad y depresión. El punto de corte utilizado generalmente es de 4/5 si se emplea una escala de respuesta dicotómica. El alfa obtenido para la medida global fue de 0,89 . Finalmente se elaboró cuestionario para evaluar los aspectos sociodemográficos y contextuales más relevantes en los cuidadores profesionales y familiares, como por ejemplo: las características del cuidador (género, edad, estado civil, cargas familiares), las características de la persona dependiente objeto de cuidados (edad, género, parentesco, tipo de dependencia padecida) y características asociadas al cuidado (tiempo como cuidador, número de personas dependientes atendidas, número de horas diarias dedicadas al cuidado, etc.) y otras variables como percepción de salud y autocuidados, o calidad de vida (entendida esta 
como satisfacción con la vida personal, laboral, familiar y social) antes y después de los cuidados. En este caso concretamente, se pedía a las personas cuidadoras que valorasen en una escala del 1 (puntuación mínima) al 10 (puntuación máxima) su grado de satisfacción y/o calidad de vida.

\section{Procedimiento}

Para obtener la muestra de cuidadores familiares y profesionales, se contactó a través de teléfono y presencialmente con varias asociaciones de ayuda mutua y empresas prestadoras del servicio de ayuda a domicilio ubicadas en Castilla y León. Una vez establecido dicho contacto, los miembros del equipo de investigación acordaron con los responsables de las entidades el procedimiento de entrega de cuestionarios. En la mayor parte de los casos, tanto las asociaciones de ayuda mutua como las empresas de ayuda a domicilio, distribuían los cuestionarios a los cuidadores y posteriormente los remitían por correo postal al equipo. En todos los casos, los cuestionarios iban en sobre cerrado, garantizando así el anonimato y la confidencialidad de los participantes. Se repartieron un total de 700 cuestionarios para la evaluación de los cuidadores profesionales, de los cuales se recogieron un total de 397 debidamente cumplimentados lo que supuso una tasa de participación del 56,71\%; y un total de 435 cuestionarios en el caso de los cuidadores familiares, de los cuales se recogieron 203 debidamente cumplimentados lo que supuso, en este caso, una tasa de participación del 46,66\%. El porcentaje total de participación en el estudio se sitúo en el $52,86 \%$. En todos los casos se mantuvo como criterio de inclusión: que el cuidador/a fuese mayor de 18 años, que en el momento de la evaluación se encontrase atendiendo y/o trabajando con una persona mayor en situación de dependencia y que llevase desempeñando dicha función durante al menos 6 meses. La muestra final quedó conformada por 600 cuidadores que accedieron a participar voluntariamente en este estudio. A todos ellos se les garantizó la confidencialidad y el anonimato en las respuestas emitidas. Los datos se recogieron a lo largo del año 2012.

\section{RESULTADOS}

Los datos fueron analizados con el programa estadístico SPSS v. 15 (2006); el nivel de confianza establecido fue de $\alpha=0,05$. En primer lugar y con objeto de dar respuesta a nuestro primer objetivo, en la Tabla 1 se presentan la distribución respecto a los niveles de carga experimentados por los cuidadores profesionales. La puntuación media obtenida en este grupo de cuidadores es de 22,88 puntos $(D T=10,26)$, lo que indica una ausencia de sobrecarga en estos trabajadores. Como puede observarse, tan sólo 11 cuidadores de este grupo experimental algún grado de sobrecarga derivado del cuidado. Respecto al grupo de cuidadores familiares, la puntuación media obtenida por este grupo fue de 40,18 puntos $(D T=16,09)$, lo que indica que a nivel general los 
cuidadores no experimentan situaciones de estrés crónico por el cuidado del familiar (puntuaciones inferiores a 46 denotan ausencia de sobrecarga). Pese a esto, 70 (34,5\%) cuidadores familiares presentan niveles de carga leve e intensa como consecuencia de los cuidados que ofrecen a su familiar dependiente (véase tabla 1).

Tabla 1. Niveles de carga experimentados por los cuidadores profesionales y familiares

\begin{tabular}{lcccc}
\hline \multirow{2}{*}{ Carga Subjetiva } & \multicolumn{2}{c}{ Cuidadores Profesionales } & \multicolumn{2}{c}{ Cuidadores Familiares } \\
\cline { 2 - 5 } & $N$ & $\%$ & $N$ & $\%$ \\
\hline Ausencia de Carga & 374 & 94,2 & 133 & 65,5 \\
\hline Sobrecarga Leve & 5 & 1,3 & 32 & 15,8 \\
\hline Sobrecarga Intensa & 6 & 1,5 & 38 & 18,7 \\
\hline
\end{tabular}

Con objeto de dar respuesta a nuestro segundo objetivo, a continuación se presentan los resultados tras el análisis de las posibles semejanzas y diferencias entre los grupos de cuidadores (profesionales vs. familiares), en función de la salud física y psicológica. Así, en la tabla 2 se observa la existencia de diferencias significativas en todas las variables de salud. En este sentido, los cuidadores familiares puntúan significativamente superior en todas las variables, lo indica que precisamente este grupo tiene peor salud.

Tabla 2. Estadísticos descriptivos y significación de las diferencias (Anova) en la salud biopsicosocial en función del tipo de cuidador

\begin{tabular}{llllll}
\hline & & $N$ & Media & D.T. & $F$ \\
\hline \multirow{2}{*}{ Síntomas Somáticos } & Cuidador Familiar & 203 & 4,15 & 2,11 & \multirow{2}{*}{$14,362^{* * *}$} \\
\cline { 2 - 5 } & Cuidador Profesional & 390 & 3,43 & 2,24 & \multirow{2}{*}{$52,883 * *$} \\
\hline \multirow{2}{*}{ Ansiedad-Insomnio } & Cuidador Familiar & 203 & 5,39 & 1,95 & \multirow{2}{*}{$99,531^{* *}$} \\
\cline { 2 - 5 } & Cuidador Profesional & 392 & 3,97 & 2,40 & \\
\hline \multirow{2}{*}{ Disfunción Social } & Cuidador Familiar & 203 & 2,28 & 1,61 & \multirow{2}{*}{$40,757 * *$} \\
\cline { 2 - 5 } Depresión & Cuidador Profesional & 393 & 1,30 & 0,78 & 1,69 \\
\hline
\end{tabular}

** Significativo con $p<0,01$

Figura 1. Salud percibida en función del tipo de cuidador (Fuente: elaboración propia)

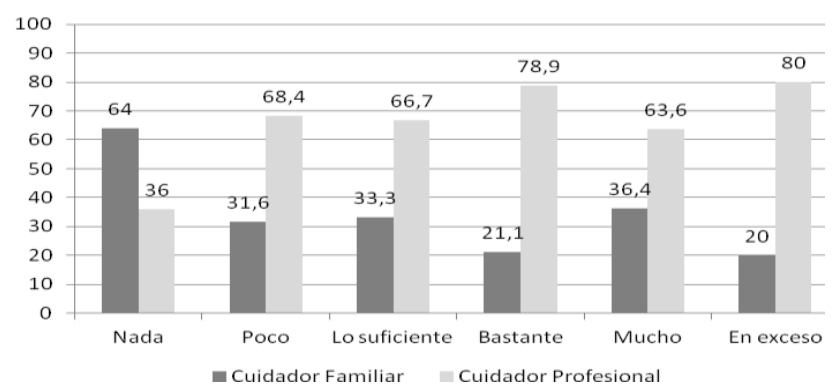

Por su parte, el análisis de la asociación entre las diferentes categorías de la percepción de cuidarse a sí mismo según el tipo de cuidador (profesional vs. familiar) 
puso de manifiesto la existencia de una asociación significativa $\left(\chi^{2}=25,616 ; d f=5\right.$; $p=0,000)$, en el sentido de que los cuidadores familiares perciben que se cuidan menos que los cuidadores profesionales (véase figura 1).

Finalmente, en la tabla 3 se presenta el análisis respecto a las posibles semejanzas y diferencias según el tipo de cuidador en las variables calidad de vida, calidad de vida laboral, calidad de vida familiar y calidad de vida social una vez se ha asumido el cuidado de la persona mayor dependiente, esto es, en el momento actual. En este caso, el grupo de cuidadores profesionales obtuvo puntuaciones significativamente más elevadas en todas las variables analizadas.

Tabla 3. Estadísticos descriptivos y significación de las diferencias (Anova) en calidad de vida, calidad de vida laboral, familiar y social en el momento actual según el tipo de cuidador (profesional vs. familiar)

\begin{tabular}{|c|c|c|c|c|c|}
\hline & & $N$ & $M$ & D.T. & $F$ \\
\hline \multirow{2}{*}{ Calidad de Vida } & Cuidador Familiar & 203 & 5,47 & 2,21 & \multirow{2}{*}{$131,254 * *$} \\
\hline & Cuidador Profesional & 365 & 7,30 & 1,56 & \\
\hline \multirow{2}{*}{ Calidad de Vida Laboral } & Cuidador Familiar & 188 & 4,87 & 2,72 & \multirow{2}{*}{$111,465^{* *}$} \\
\hline & Cuidador Profesional & 395 & 6,86 & 1,76 & \\
\hline \multirow{2}{*}{ Calidad de Vida Familiar } & Cuidador Familiar & 203 & 6,12 & 2,38 & \multirow{2}{*}{$95,416 * *$} \\
\hline & Cuidador Profesional & 395 & 7,82 & 1,79 & \\
\hline \multirow{2}{*}{ Calidad de Vida Social } & Cuidador Familiar & 203 & 4,82 & 2,60 & \multirow{2}{*}{$60,111 * *$} \\
\hline & Cuidador Profesional & 396 & 6,51 & 2,46 & \\
\hline
\end{tabular}

\section{CONCLUSIONES}

Los resultados obtenidos nos permiten concluir que los cuidadores familiares experimentan mayores niveles de sobrecarga como consecuencia de la atención ofrecida al familiar dependiente que aquellos que realizan estas tareas de manera profesional. La formación especializada, la remuneración recibida y la menor implicación emocional de estos cuidadores contribuirían a explicar estos resultados (Herrera, 1998). Por otro lado, también es posible evidenciar diferencias entre ambos grupos de cuidadores en lo que respecta a su salud física y psicológica. Concretamente, los resultados han puesto de manifiesto que los cuidadores familiares de personas mayores dependientes tienen una peor salud física y psicológica que los cuidadores profesionales. En este sentido experimentan una mayor sintomatología somática (p.e. dolores de cabeza, musculares) y mayores niveles de ansiedad e insomnio. Por su parte, también presentan una peor salud y manifiestan no cuidarse nada o hacerlo menos de lo que deberían en comparación con los cuidadores profesionales que se encuentran en una situación más ventajosa. Resultados similares también se han encontrado en los pocos estudios comparativos que se han llevado a cabo con ambos grupos de cuidadores (Aparicio et al., 2008; Sánchez-López, Aparicio-García y Dresch, 2006). Además, el cuidado del dependiente también repercute significativamente en los familiares provocándoles una menor satisfacción con la vida en general y con otros factores como el trabajo, familia y las 
relaciones sociales. Sin embargo, para los cuidadores profesionales ocurre totalmente lo contrario, esto es, los cuidados del dependiente hacen que experimenten una mayor satisfacción con la vida, con el trabajo, con la familia y con las relaciones sociales. Explicaciones potenciales a estos resultados pueden ser las características específicas del colectivo de ayuda a domicilio. Esto es, el cuidado de personas dependientes constituye una alternativa laboral remunerada que, pese a las dificultades intrínsecas de la tarea, permite a estos profesionales mejorar sus condiciones de vida. No obstante, se precisarán de otros estudios que confirmen o refuten esta hipótesis. Finalmente como se ha puesto de manifiesto en este trabajo existen diferencias en la salud y la calidad de vida experimentada por ambos grupos de cuidadores, por lo que se debe ser cuidadoso y no valorar conjuntamente a estos dos grupos, como se ha venido haciendo en la mayoría de las investigaciones realizadas con cuidadores (Aparicio et al., 2008). Por otro lado, es necesario poner en marcha intervenciones, especialmente en el sector de los cuidadores familiares, a través de la realización de cursos y programas psicoeducativos (p.e. inoculación de estrés) donde se les enseñe a los cuidadores estrategias para hacer frente a los cuidados del familiar dependiente y mejorar así su salud.

\section{Agradecimientos}

Investigación parcialmente financiada por la Consejería de Educación de la Junta de Castilla y León (España) (Referencia: SA008A08).

\section{REFERENCIAS}

Aparicio, M.E., Sánchez, M.P., Díaz, J.F., Cuéllar, I., Castellanos, B., Fernández, M.T. y de Tena, A. (2008). La salud física y psicológica de las personas cuidadoras: comparación entre cuidadores formales e informales. Madrid: IMSERSO.

Brummett, B.H., Babyak, M.A., Siegler, I.C., Vitaliano, P., Ballard, E.L., Gwyther, L.P., y Williams, R.B. (2006). Associations among perceptions of social support, negative affect, and quality of sleep in caregivers and noncaregivers. Health Psychology, 25, 220225.

Crespo, M. y López, J. (2007). El apoyo a los cuidadores de familiares mayores dependientes en el hogar: desarrollo del programa "Cómo mantener su bienestar". Madrid: IMSERSO.

Feldberg, C., Tartaglini, M.F., Clemente, M.A., Petracca, G., Cáceres, F. y Stefani, D. (2011).Vulnerabilidad psicosocial del cuidador familiar. Creencias acerca del estado de salud del paciente neurológico y el sentimiento de sobrecarga. Neurología Argentina, 3(1), 11-17.

Fernández de Larrinoa, P., Martínez, S., Ortiz, S., Carrasco, M., Solabarrieta, J. y Gómez, I. (2011). Autopercepción del estado de salud en familiares cuidadores y su relación con el nivel de sobrecarga. Psicothema, 23(3), 388-393.

Flores, N., Jenaro, C., Navarro, E., Vega, V. y Vázquez, A. (2013). Salud biopsicosocial y relación con la carga experimentada por cuidadores familiares de personas mayores dependientes. Revista Ibero-Americana de Gerontología (RIAG), 1, 47-60. 
Francisco del Rey, J.C. y Mazarraza, L. (1995). Cuidados informales. Revista Rol de Enfermería, 202, 61-65.

Goldberg, D.P. y Hillier, V.F. (1979). A scaled version of the General Health Questionnaire. Psychological Medicine, 9, 139-145.

Grandón, P., Jenaro, C. y Lemos, S. (2008). Primary caregivers of schizophrenia outpatiens: Burden and predictor variables. Psychiatry Research, 158, 335-343.

Herrera-Gómez, M. (1998). Nuevos desvaríos en políticas sociales: la community care. Revista Española de Investigaciones Sociológicas, 82, 249-281.

Hartke, R.J., King, R.B., Heinemann, A.W. y Semik, P. (2006). Accidents in older caregivers of persons surviving stroke and their relation to caregiver stress. Rehabilitation Psychology,51, 150-156.

IMSERSO (2005). Cuidados a las personas mayores en los hogares españoles. El entorno familiar. Madrid: IMSERSO.

Jenaro, C., Flores, N. y Arias, B. (2007). Burnout and Coping in Human Service Practitioners. Professional Psychology: Research and Practice, 38, 80-87.

Ley 39/2006, de 14 de diciembre de Promoción de la Autonomía Personal y Atención a las personas en situación de dependencia. BOE núm 299 de 15 de diciembre.

Lobo, A., Pérez, M.J. y Artal, J. (1989). Validity of the scaled version of the General Health Questionnarie (GHQ 28) in a Spanish population. Psychological Medicine, 16(1), 135140.

Maslach, C., Schaufeli, W.B. y Leiter, M.P. (2001). Job Burnout. Annuary Review Psychology, 52, 397-422.

Menezes de Lucena, V., Fernández, B., Hernández, L., Ramos, F. y Contador, I. (2006). Resiliencia y modelo de burnout-engagement en cuidadores formales de ancianos. Psicothema, 18, 791-796.

Rivera, J. (2001). Redes familiares en el cuidado del anciano con demencia. Análisis evolutivo de un estudio poblacional. Madrid: Consejo Económico y Social.

Rogero, J. (2010). Los tiempos del cuidado. El impacto de la dependencia de los mayores en la vida cotidiana de sus cuidadores. Madrid: Instituto de Mayores y Servicios Sociales (IMSERSO).

Ruíz-Robledillo, N. y Moya-Albiol, L. (2012). El cuidado informal: una visión actual. Revista de Motivación y Emoción, 1, 22-30.

Sánchez-López, M.P., Aparicio-García, M.E. y Dresch, V. (2006). Ansiedad, autoestima y satisfacción autopercibida como predictores de salud: diferencias entre hombres y mujeres. Psicothema, 18(3), 583-589.

SPSS, Inc. (2006). SPSS base 15 for windows user's guide. Chicago, IL: SPSS Inc.

Zarit, S.H., Reever, K.E. y Bach-Peterson, J. (1980). Relatives of the impaired elderly: Correlates of feeling of burden. Gerontologist, 20, 646-655.

Recibido: 7 de abril de 2014

Recepción Modificaciones: 26 de mayo de 2014

Aceptado: 1 de junio de 2014 